$\mathrm{J}$ o u r n a l of

Mathematics

and Applications

No 35, pp 91-95 (2012)

\title{
Complete convergence under special hypotheses
}

\author{
G. Stoica
}

Submitted by: Jan Stankiewicz

ABstract: We prove Baum-Katz type theorems along subsequences of random variables under Komlós-Saks and Mazur-Orlicz type boundedness hypotheses

AMS Subject Classification: 60F15, 60E15

Key Words and Phrases: Complete convergence, boundedness hypotheses, law of large numbers

\section{Introduction and main results}

Throughout the paper we shall work with real valued random variables on a complete probability space $(\Omega, \mathcal{F}, P)$. The following Baum-Katz type result (cf. [5]) quantifies the rate of convergence in the strong law of large numbers for general sequences of random variables in the form of a complete convergent series:

Theorem 0. If $\left(X_{n}\right)_{n \geq 1}$ is an $L^{p}$-norm bounded sequence for some $0<p<2$, i.e., $\sup _{n \geq 1}\left\|X_{n}\right\|_{p} \leq C$ for some $C>0$, then there exists a subsequence $\left(Y_{n}\right)_{n \geq 1}$ of $\left(X_{n}\right)_{n \geq 1}$ such that, for all $0<r \leq p$, we have

$$
\sum_{n=1}^{\infty} n^{p / r-2} P\left(\left\{\omega \in \Omega:\left|\sum_{j=1}^{n} Y_{j}(\omega)\right|>\varepsilon n^{1 / r}\right\}\right)<\infty \text { for } \varepsilon>0
$$

In particular the strong law of large numbers holds along the subsequence $\left(Y_{n}\right)_{n \geq 1}$, i.e., $Y_{n} / n^{1 / p} \rightarrow 0$ a.s.

The examples in [6], [4] and [3] show that (1) may fail if one drops the $L^{p}$-norm boundedness hypothesis. Inspired by the celebrated Komlós-Saks and Mazur-Orlicz extensions of the law of large numbers, in this note we shall prove two versions of

COPYRIGHT (c) by Publishing Department Rzeszów University of Technology P.O. Box 85, 35-959 Rzeszów, Poland 
the Baum-Katz theorem under special boundedness hypotheses, more general than $L^{p}$-norm boundedness condition required in Theorem 0 .

Theorem 1. Let $0<p<2$ and $\left(X_{n}\right)_{n \geq 1}$ a sequence such that $\lim \sup _{n}\left|X_{n}(\omega)\right|^{p}<\infty$ for all $\omega \in \Omega$. Then there exists a subsequence $\left(Y_{n}\right)_{n \geq 1}$ of $\left(X_{n}\right)_{n \geq 1}$ such that (1) holds for all $0<r \leq p$.

Theorem 2. Let $0<p<2$ and $\left(X_{n}\right)_{n \geq 1}$ a sequence satisfying the following condition: for every subsequence $\left(\tilde{X}_{n}\right)_{n \geq 1}$ of $\left(X_{n}\right)_{n \geq 1}$ and $n \geq 1$, there exists a convex combination $Z_{n}$ of $\left\{\left|\tilde{X}_{n}\right|^{p},\left|\tilde{X}_{n+1}\right|^{p}, \ldots\right\}$, such that $\lim \sup _{n}\left|Z_{n}(\omega)\right|<\infty$ for all $\omega \in \Omega$. Then there exists a subsequence $\left(Y_{n}\right)_{n \geq 1}$ of $\left(X_{n}\right)_{n \geq 1}$ such that (1) holds for all $0<r \leq p$.

Remarks. (i) Both Theorems 1 and 2 hold for uniformly bounded sequences $\left(X_{n}\right)_{n \geq 1}$ in $L^{p}, 0<p<2$. On $[0,1]$ endowed with the Lebesgue measure, the sequence $X_{n}(\omega)=$ $n^{2}$ if $0 \leq \omega \leq 1 / n$ and 0 otherwise, satisfies Theorem 2 because $X_{n} \rightarrow 0$ Lebesgue-a.s., yet it does not satisfy Theorem 1 with $p=1$ because it is not bounded in $L^{1}[0,1]$. As a matter of fact, both Theorems 1 and 2 may fail for unbounded sequences, e.g., $X_{n}=n$.

(ii) The idea beneath Theorems 1 and 2 is to construct a rich family of uniformly integrable subsequences of $\left(X_{n}\right)_{n \geq 1}$ as in [2], for which condition (1) holds; note that the hypotheses in [6] and [3] cannot produce Baum-Katz type theorems, as the families of subsequences therein are no longer uniformly integrable.

\section{Proofs of the results}

Proof of Theorem 1. Note that $\lim \sup _{n}\left|X_{n}(\omega)\right|^{p}<\infty$ is equivalent to

$$
\sup _{n \geq 1}\left|X_{n}(\omega)\right|^{p}<\infty
$$

for all $\omega \in \Omega$. For any natural number $m \geq 1$, let us define

$$
A_{m}=\left\{\omega \in \Omega: \sup _{n \geq 1}\left|X_{n}(\omega)\right|^{p} \leq m\right\}
$$

Assume that $r<p$ and fix $a>p / r-1$. As $P\left(A_{m}\right) \rightarrow 1$ as $m \rightarrow \infty$, we can choose $m_{1} \geq 1$ such that $P\left(A_{m_{1}}\right)>1-2^{-a}$. Integrating and applying Fatou's lemma, we obtain

$$
\sup _{n \geq 1} \int_{A_{m_{1}}}\left|X_{n}(\omega)\right|^{p} d P(\omega) \leq m_{1} .
$$

We now apply the Biting Lemma (cf. [1]) to the sequence $\left(X_{n}\right)_{n \geq 1}$ and obtain: an increasing sequence of sets $\left(B_{k}^{1}\right)_{k \geq 1}$ in $\mathcal{F}$ with $P\left(B_{k}^{1}\right) \rightarrow 1$ as $k \rightarrow \infty$, and a subsequence $\left(X_{n}^{1}\right)_{n \geq 1}$ of $\left(X_{n}\right)_{n \geq 1}$ such that $\left(X_{n}^{1}\right)_{n \geq 1}$ is uniformly integrable on each 
set $A_{m_{1}} \cap B_{k}^{1}, k \geq 1$. The latter fact together with estimate (2) show that Theorem 0 applies to the sequence $\left(X_{n}^{1}\right)_{n \geq 1}$ and gives

$$
\sum_{n=1}^{\infty} n^{p / r-2} P\left(\left\{\omega \in A_{m_{1}} \cap B_{k}^{1}:\left|\sum_{j=1}^{n} X_{j}^{1}(\omega)\right|>\varepsilon n^{1 / r}\right\}\right)<\infty \text { for } \varepsilon>0 \text { and } k \geq 1 .
$$

Another application of the Biting Lemma to $\left(X_{n}^{1}\right)_{n \geq 1}$, instead of $\left(X_{n}\right)_{n \geq 1}$, produces: a measurable set $A_{m_{2}}$ with $P\left(A_{m_{2}}\right)>1-3^{-a}$, an increasing sequence of sets $\left(B_{k}^{2}\right)_{k \geq 1}$ in $\mathcal{F}$ with $P\left(B_{k}^{2}\right) \rightarrow 1$ as $k \rightarrow \infty$, and a subsequence $\left(X_{n}^{2}\right)_{n \geq 1}$ of $\left(X_{n}^{1}\right)_{n \geq 1}$ such that $\left(X_{n}^{2}\right)_{n \geq 1}$ is uniformly integrable on each set $A_{m_{2}} \cap B_{k}^{2}, k \geq 1$, such that

$$
\sum_{n=1}^{\infty} n^{p / r-2} P\left(\left\{\omega \in A_{m_{2}} \cap B_{k}^{2}:\left|\sum_{j=1}^{n} X_{j}^{2}(\omega)\right|>\varepsilon n^{1 / r}\right\}\right)<\infty \text { for } \varepsilon>0 \text { and } k \geq 1 .
$$

By induction, we construct for each $i \geq 1$ : a measurable set $A_{m_{i}}$ with $P\left(A_{m_{i}}\right)>$ $1-(i+1)^{-a}$, an increasing sequence of sets $\left(B_{k}^{i}\right)_{k \geq 1}$ in $\mathcal{F}$ with $P\left(B_{k}^{i}\right) \rightarrow 1$ as $k \rightarrow \infty$, and a subsequence $\left(X_{n}^{i}\right)_{n \geq 1}$ of $\left(X_{n}^{i-1}\right)_{n \geq 1}$, with the convention that $\left(X_{n}^{0}\right)_{n \geq 1}$ is precisely $\left(X_{n}\right)_{n \geq 1}$, such that $\left(X_{n}^{i}\right)_{n \geq 1}$ is uniformly integrable on each set $A_{m_{i}} \cap B_{k}^{i}$, $k \geq 1$, and

$$
\sum_{n=1}^{\infty} n^{p / r-2} P\left(\left\{\omega \in A_{m_{i}} \cap B_{k}^{i}:\left|\sum_{j=1}^{n} X_{j}^{i}(\omega)\right|>\varepsilon n^{1 / r}\right\}\right)<\infty \text { for } \varepsilon>0 \text { and } k, i \geq 1 .
$$

Now define $Y_{n}:=X_{n}^{n}$ and, using a diagonal argument in the above formula, we obtain that

$$
\sum_{n=1}^{\infty} n^{p / r-2} P\left(\left\{\omega \in A_{m_{n}} \cap B_{k}^{n}:\left|\sum_{j=1}^{n} Y_{j}(\omega)\right|>\varepsilon n^{1 / r}\right\}\right)<\infty \text { for } \varepsilon>0 \text { and } k \geq 1 .
$$

As $P\left(B_{k}^{n}\right) \rightarrow 1$ as $k \rightarrow \infty$ for all $n \geq 1$, formula (3) and the dominated convergence theorem imply that

$$
\sum_{n=1}^{\infty} n^{p / r-2} P\left(\left\{\omega \in A_{m_{n}}:\left|\sum_{j=1}^{n} Y_{j}(\omega)\right|>\varepsilon n^{1 / r}\right\}\right)<\infty \text { for } \varepsilon>0
$$

Therefore, to prove that series (1) converges for our subsequence $\left(Y_{n}\right)_{n \geq 1}$ and $r<p$, it suffices to prove (4) with $A_{m_{n}}$ replaced by its complement, i.e.,

$$
\sum_{n=1}^{\infty} n^{p / r-2} P\left(\left\{\omega \in \Omega \backslash A_{m_{n}}:\left|\sum_{j=1}^{n} Y_{j}(\omega)\right|>\varepsilon n^{1 / r}\right\}\right)<\infty \text { for } \varepsilon>0 .
$$

Indeed, the latter series is

$$
\leq \sum_{n=1}^{\infty} n^{p / r-2} P\left(\left\{\omega \in \Omega \backslash A_{m_{n}}\right\}\right) \leq \sum_{n=1}^{\infty} n^{p / r-2-1}<\infty
$$


as $P\left(A_{m_{n}}\right)>1-(n+1)^{-a}>1-n^{-a}$ and $a>p / r-1$. The proof is achieved in the case $r<p$.

If $r=p$, then we modify the induction process as follows: choose measurable sets $A_{m_{i}}$ with $P\left(A_{m_{i}}\right)>i /(i+1)$ for all $i \geq 1$; as such, the diagonal argument above gives the following replacement of (4):

$$
\sum_{n=1}^{\infty} \frac{1}{n}\left(\left\{\omega \in A_{m_{n}}:\left|\sum_{j=1}^{n} Y_{j}(\omega)\right|>\varepsilon n^{1 / r}\right\}\right)<\infty \text { for } \varepsilon>0 .
$$

To show that series (1) converges for our subsequence $\left(Y_{n}\right)_{n \geq 1}$ and $r=p$, it suffices to prove the following replacement of (5):

$$
\sum_{n=1}^{\infty} \frac{1}{n} P\left(\left\{\omega \in \Omega \backslash A_{m_{n}}:\left|\sum_{j=1}^{n} Y_{j}(\omega)\right|>\varepsilon n^{1 / r}\right\}\right)<\infty \text { for } \varepsilon>0 .
$$

Indeed, the latter series is

$$
\leq \sum_{n=1}^{\infty} \frac{1}{n} P\left(\left\{\omega \in \Omega \backslash A_{m_{n}}\right\}\right) \leq \sum_{n=1}^{\infty} \frac{1}{n(n+1)}<\infty
$$

by the choice of $P\left(A_{m_{n}}\right), n \geq 1$. The latter is the substitute of (6) in the case $r=p$, and the proof is now complete.

Proof of Theorem 2. By hypothesis we can write

$$
Z_{n}=\sum_{i \in I_{n}} \lambda_{i}^{n}\left|\tilde{X}_{n+i}\right|^{p} \text { for some } \lambda_{i}^{n} \geq 0 \text { with } \sum_{i \in I_{n}} \lambda_{i}^{n}=1,
$$

and where $I_{n}$ are finite subsets of $\{0,1,2, \ldots\}$. In addition, the sequence $\left(Z_{n}\right)_{n \geq 1}$ satisfies the condition $\sup _{n \geq 1}\left|Z_{n}(\omega)\right|^{p}<\infty$ for all $\omega \in \Omega$. For any natural number $m \geq 1$, let us define $A_{m}=\left\{\omega \in \Omega: \sup _{n \geq 1}\left|Z_{n}(\omega)\right| \leq m\right\}$. As $P\left(A_{m}\right) \rightarrow 1$ as $m \rightarrow \infty$, we can choose $m_{1} \geq 1$ such that $P\left(A_{m_{1}}\right)>1-2^{-a}$ or $1 / 2$, according to $p>r$ or $p=r$, and where $a>p / r-1$ is fixed. Integrating and applying Fatou's lemma, we obtain

$$
\sup _{n \geq 1} \sum_{i \in I_{n}} \lambda_{i}^{n} \int_{A_{m_{1}}}\left|\tilde{X}_{n+i}(\omega)\right|^{p} d P(\omega) \leq m_{1} .
$$

Hence there is a subsequence $\left(\bar{X}_{n}\right)_{n \geq 1}$ of $\left(\tilde{X}_{n}\right)_{n \geq 1}$ (therefore of $\left(X_{n}\right)_{n \geq 1}$ as well), such that

$$
\sup _{n \geq 1} \int_{A_{m_{1}}}\left|\bar{X}_{n}(\omega)\right|^{p} d P(\omega) \leq m_{1}
$$

which is precisely eq. (2) along a subsequence. The remainder of the proof goes exactly as in the proof of Theorem 1 . 


\section{References}

[1] 1 J.K. Brooks, R.V. Chacon, Continuity and compactness of measures, Adv. Math. 37 (1980) 16-26.

[2] C. Castaing, M. Saadoune, Komlós type convergence for random variables and random sets with applications to minimization problems, Adv. Math. Econ. 10 (2007) 1-29.

[3] S.J. Dilworth, Convergence of series of scalar-and vector-valued random variables and a subsequence principle in $L_{2}$, Trans. Amer. Math. Soc. 301 (1987) 375-384.

[4] E. Lesigne, D. Volný, Large deviations for martingales, Stoch. Proc. Appl. 96 (2001) 143-159.

[5] G. Stoica, The Baum-Katz theorem for bounded subsequences, Stat. Prob. Lett. 78 (2008), 924-926.

[6] H. von Weizsäcker, Can one drop the $L^{1}$-boundedness in Komlós' subsequence theorem? Amer. Math. Monthly 111 (2004) 900-903.

DOI: $10.7862 / \mathrm{rf.2012.8}$

\section{George Stoica}

email: stoica@unb.ca

Department of Mathematical Sciences,

University of New Brunswick,

Saint John NB, Canada

Received 17.12.2010, Revisted 2\%.10.2011 